\title{
Effect of polydispersity, bimodality and aspect ratio on the phase behavior of colloidal platelet suspensions
}

\author{
Yuri Martínez-Ratón \\ Grupo Interdisciplinar de Sistemas Complejos (GISC), Departamento de Matemáticas, \\ Escuela Politécnica Superior, Universidad Carlos III de Madrid, \\ Avenida de la Universidad 30, E-28911, Leganés, Madrid, Spain \\ Enrique Velasco \\ Departamento de Física Teórica de la Materia Condensada and Instituto de Ciencia de Materiales Nicolás Cabrera, \\ Universidad Autónoma de Madrid, E-28049 Madrid, Spain
}

(Dated: September 20, 2018)

\begin{abstract}
We use a Fundamental-Measure density functional for hard board-like polydisperse particles, in the restricted-orientation approximation, to explain the phase behaviour of platelet colloidal suspensions studied in recent experiments. In particular, we focus our attention on the behavior of the total packing fraction of the mixture, $\eta$, in the region of two-phase isotropic-nematic coexistence as a function of mean aspect ratio, polydispersity and fraction of total volume $\gamma$ occupied by the nematic phase. In our model, platelets are polydisperse in the square section, of side length $\sigma$, but have constant thickness $L$ (and aspect ratio $\kappa \equiv L /\langle\sigma\rangle<1$, with $\langle\sigma\rangle$ the mean side length). Good agreement between our theory and recent experiments is obtained by mapping the real system onto an effective one, with excluded volume interactions but with thicker particles (due to the presence of long-ranged repulsive interactions between platelets). The effect of polydispersity in both shape and particle size has been taken into account by using a size distribution function with an effective mean-square deviation that depends on both polydispersities. We also show that the bimodality of the size distribution function is required to correctly describe the huge two-phase coexistence gap and the nonlinearity of the function $\gamma(\eta)$, two important features that these colloidal suspensions exhibit.
\end{abstract}

PACS numbers: 61.30.Cz, 61.30.Hn, 61.20.Gy

\section{INTRODUCTION}

Colloidal suspensions of mineral or viral anisotropic particles interacting via short-ranged repulsive forces exhibit a phase behavior with entropy-driven phase transitions between their liquid-crystalline phases. The nature of these phases strongly depends on particle geometry. The rod geometry in mineral or viral particles favours the formation of uniform phases, i.e. isotropic (I) and nematic $(\mathrm{N})$ phases [1], and also of the layered smectic (S) phase 2-4]. In the case of the plate geometry, the I and $\mathrm{N}$ phases are usually stabilized at low particle concentration [5], and the $\mathrm{N}$ phase requires a relatively high aspect ratio (and thus particle anisotropy) [5]. In addition, as the volume fraction increases, there may appear a transition to the columnar (C) phase [6, 7].

The plate geometry is more versatile as regards the type of liquid crystalline phases it may induce [8]. Recently it has been shown that colloidal suspensions of some plate-like, mineral charged particles, can also stabilize the smectic phase [9, 10]. The colloidal particles are usually polydisperse in their sizes (diameter and thickness) and shapes (rod or plate geometry or different particle cross-sections), and it was found that polydispersity causes phase behavior in these systems to be much more complex due to phenomena such as size segregation, fractionation and multiple phase coexistence [11]. For example, polydisperse rod-plate mixtures exhibit demixing between $\mathrm{I}$ and different $\mathrm{N}$ phases (with the former populated by particles with less anisotropy), and also up to four coexisting phases (some of them nonuniform) may exist at high densities [11]. No trace of biaxial N phases was found in these mixtures. However, recent experiments on board-like colloidal particles could find this elusive phase [12]. Also I-N coexistence with density inversion has been observed [13, 14] (with the I phase being the densest phase).

The theoretical modeling of these kind of mixtures turns out to be a difficult task. Density functional theory (DFT), which is based on the local density distribution, has been successful in the description of bulk and interfacial phase behavior of hard spheres and other fluids of anisotropic particles [15], but it becomes hard to implement for the case of polydisperse mixtures. This is due to the huge number of degrees of freedom on which the local density now depends: not only on spatial and (for anisotropic particles) orientational coordinates, but also on a number of polydispersity variables. Despite the increased difficulty, some theoretical calculations on polydisperse mixtures of freely rotating hard rods in the Onsager approximation have been performed [16, 17]. These calculations confirm the phase behavior found in experiments as regards the broadening of the I-N coexisting gap and the size-fractionation phenomenon. In this respect, it would be interesting to extend the recently proposed Fundamental-Measure DFT for freely rotating hard disks [18] to the calculation of phase behavior in polydisperse platelets. 
Monte Carlo simulations on polydisperse infinitely thin hard-platelet fluids have also been carried out [19]. These results again show the dramatic effect of polydispersity on phase behavior in hard-platelet fluids (mainly segregation driven by particle size), as compared to that in one-component fluids, already simulated in the 90 s for the cut-sphere geometry [20]. In the latter work the bulk phase diagram was traced. For aspect ratios $\kappa \equiv L / \sigma<1$ the $\mathrm{N}$ and, at higher densities the $\mathrm{C}$, phases were found to be stable (here $L$ is the thickness and $\sigma$ the particle diameter). In the range $0.1 \lesssim \kappa \lesssim 0.2$ the $\mathrm{C}$ phase gives rise to the I and eventually for $\kappa \sim 0.2$ to the cubatic phase, and finally for $\kappa \gtrsim 0.3$ only the I and the solid phase are stable. Similar phase behavior was found in Monte Carlo simulations of hard oblate spherocylinders [21], where two different crystals are stable (tilted for $\kappa \lesssim 0.45$ and aligned for $\kappa \gtrsim 0.45$ ); the cubatic phase is always unstable. The practical difficulties in implementing DFT calculations associated with polydispersity can be circumvented by considering discrete particle orientations, as in the Zwanzig model where the main axes of the particles, one for uniaxial and two for biaxial geometries, point along one of the three Cartesian axes [22]. In the framework of this model the phase diagrams of polydisperse hard rods [23] and rod-plate mixtures [24] have been calculated.

Within the same approach, the effect of polydispersity on the stability of the biaxial $\mathrm{N}$ phase of hard board-like biaxial particles [25] has recently been studied. The phase diagram of the one-component limit of this fluid was recently obtained [26] using a DFT based on Fundamental-Measure theory for hard biaxial parallelepipeds [27]. Finally, the same model has been applied to the study of interfacial properties of binary mixtures confined by external potentials [28].

Recently a systematic experimental study of the phase behaviour of polydisperse platelets in suspension has been presented [30]. Particles were synthesized by hydrothermal methods, and further exfoliation (through chemical treatment with TBA molecules the pristine Zirconium-Phosphate (ZrP) crystals are delaminated into single layers) [30]. The novel feature of this fluid is that polydispersity is in the platelet diameter, with a strictly constant thickness. Also the shape of the particle cross sections is polydisperse, with most particles having hexagonal geometry. The work was focused on the study of the I-N transition for different polydispersities and mean aspect ratios. The aim of the present article is to theoretically understand the results presented in Ref. [30]. The experimental results and the conclusions we obtain from our theoretical model can be summarized as follows:

(i) Highly polydisperse platelet mixtures exhibit a huge I-N coexistence gap which cannot be explained by simply assuming a unimodal distribution function for particle diameters. However, if one considers a bimodal distribution function, the coexistence gap can be explained via a demixing mechanism. We remark that bimodality may not be apparent through a direct visual inspection of the bimodal distribution function. Note that the effect of bimodality in platelet thickness (not diameter) on phase behavior has already been studied in Ref. [14], but in that case the bimodality in the size distribution function is clearly seen from the size histogram, which is not the case in Ref. [30].

(ii) The repulsive character of colloidal platelet interactions in the experimental system is incorporated through an effective platelet thickness $L_{\text {eff }}$ which is much higher than the thickness of the real platelets. The effective thickness is chosen to guarantee a reasonable description of experimental data by the theoretical model.

(iii) In order to account for the shape polydispersity and to adequately describe the experimental findings, the polydispersity coefficient (mean square deviation of the size distribution function) should be taken much higher than that given in Ref. [30]. In the present study we have used a DFT for hard board-like polydisperse particles with square cross sections of width $\sigma$ and constant thickness $L$, in the oblate particle regime $\kappa=L / \sigma<1$. The DFT is based on the FMT for the hard-parallelepipeds in the restricted-orientation approximation [27].

The article is organized as follows. In Sec. II we present the theoretical model, making special emphasis on the implementation of the I-N coexistence calculations (Sec. IA), the size distribution function used to model polydispersity (Sec. IIB ) and the effect of particle shape polydispersity on the effective size polydispersity of the mixture (Sec. IIC). Sec. III presents the results, and is divided in two sections: Sec. IIIA which is devoted to the phase behavior of the mixture assuming a unimodal size distribution for diameters, and Sec. IIB. which presents results obtained with bimodal distributions. Finally we draw some conclusions in Sec. IV

\section{THEORY}

The theory we use is based on a density functional for hard board-like particles, formulated in the restricted orientation approximation (the so-called Zwanzig model). Particles are polydisperse in the side length $\sigma$ of the square section, but their thickness $L$ is fixed. The mean aspect ratio $\kappa \equiv L /\langle\sigma\rangle$ (with $\langle\sigma\rangle$ the mean side length) is less than unity, $\kappa<1$, so that we are in the oblateparticle regime. The main quantities that describe our model are the set of density distribution functions $\rho_{\nu}(\sigma)$, where $\nu=\{x, y, z\}$ refers to particles with their main axis parallel to the $\nu$ Cartesian axis; each of the three orientations can be considered to correspond to a different species, and the fluid can therefore be treated as a three-species mixture. In the following sections we describe the theoretical formalism we have used to calculate the isotropic (I)-nematic ( $\mathrm{N})$ coexistence in polydisperse mixtures that possess unimodal/bimodal size distributions. 


\section{A. Coexistence calculations}

If a fraction $\gamma$ of the total volume of the system $V$ is occupied by a nematic $(\mathrm{N})$ phase in coexistence with the isotropic (I) phase, then the density distribution functions $\rho_{\nu, s}(\sigma)$ of the two phases, $s=\{\mathrm{I}, \mathrm{N}\}$ should fulfill the lever rule (conservation of the total number of particles):

$$
\rho_{0}(\sigma)=\gamma \sum_{\nu} \rho_{\nu, \mathrm{N}}(\sigma)+(1-\gamma) \sum_{\nu} \rho_{\nu, \mathrm{I}}(\sigma) .
$$

The density distributions of all species are the same for the I phase, $\rho_{x, \mathrm{I}}(\sigma)=\rho_{y, \mathrm{I}}(\sigma)=\rho_{z, \mathrm{I}}(\sigma) \equiv \rho_{\mathrm{I}}(\sigma)$, while for the uniaxial $\mathrm{N}$ phase we have $\rho_{x, \mathrm{~N}}(\sigma)=\rho_{y, \mathrm{~N}}(\sigma) \equiv$ $\rho_{\perp, \mathrm{N}}(\sigma)$ and $\rho_{z, \mathrm{~N}}(\sigma) \equiv \rho_{\|, \mathrm{N}}(\sigma)$; here we take the nematic director to be parallel to the $z$-axis. The density distribution function $\rho_{0}(\sigma)=\rho_{0} h(\sigma)$ (parent distribution function) is a product of the mean parent number density $\rho_{0}$ and the size distribution function $h(\sigma)$ which fulfills the normalization condition $\int d \sigma h(\sigma)=1$. Note that $h(\sigma)$ has units of [Length $]^{-1}$. As $\rho_{0}$ is the number density having units of [Length $]^{-3}$ the distribution functions $\rho_{0}(\sigma)$ and $\rho_{\nu, s}(\sigma)$ have units of [Lenght $]^{-4}$.

For the whole system $\mathrm{I}+\mathrm{N}$ (with $0 \leq \gamma \leq 1$ ), we define a Lagrange functional from the free-energy density in reduced thermal units, $\tilde{\Phi}=\beta F / V$ (with $F$ the free energy, $\beta=1 / k T, k$ Boltzmann constant and $T$ the temperature), as

$$
\begin{aligned}
& \tilde{\Phi}\left[\left\{\rho_{\nu, s}\right\}\right]=\gamma \Phi\left[\left\{\rho_{\nu, \mathrm{N}}\right\}\right]+(1-\gamma) \Phi\left[\left\{\rho_{\nu, \mathrm{I}}\right\}\right] \\
& +\int d \sigma \mu_{0}(\sigma)\left[\rho_{0}(\sigma)-\gamma \sum_{\nu} \rho_{\nu, \mathrm{N}}(\sigma)\right. \\
& \left.-(1-\gamma) \sum_{\nu} \rho_{\nu, \mathrm{I}}(\sigma)\right]
\end{aligned}
$$

where $\mu_{0}(\sigma)$ are the Lagrange multipliers that guarantee the constraints (1). Note that $\mu_{0}(\sigma)$ is just the scaled with $\beta$ chemical potential of species $\nu$ of width $\sigma$ in each one of the coexisting phases, i.e. $\beta \mu_{\nu, s}(\sigma)=\mu_{0}(\sigma), \forall$ $\nu=x, y, z$ and $s=\mathrm{I}, \mathrm{N}$. $\Phi$ is split in ideal

$$
\Phi_{\mathrm{id}}\left[\left\{\rho_{\nu, s}\right\}\right]=\int d \sigma \sum_{\nu} \rho_{\nu, s}(\sigma)\left[\ln \rho_{\nu, s}(\sigma)-1\right],
$$

and excess $\Phi_{\text {exc }}$ parts. In our treatment, we obtain the latter from Fundamental-Measure Theory (FMT). In the FMT formalism $\Phi_{\text {exc }}\left(\left\{\rho_{\nu, s}^{\alpha}\right\}\right)$ is a function of a finite number of moments of the distribution function. The latter are defined as

$$
\rho_{\nu, s}^{(\alpha)}=\int d \sigma \rho_{\nu, s}(\sigma) \sigma^{\alpha}, \quad, \alpha=0,1,2 .
$$

The expression for the function $\Phi_{\text {exc }}\left(\left\{\rho_{\nu, s}^{\alpha}\right\}\right)$ [see Eq. (A5)] is obtained in the Appendix from the scaled particle theory (SPT), the uniform limit of the fundamental measure free-energy density functional [27]. The constrained functional minimization of (2) with respect to $\rho_{\nu, s}(\sigma)$, together with the definition (4), provide the moments at equilibrium [23]:

$$
\rho_{\nu, s}^{(\alpha)}=\rho_{0} \int d \sigma \frac{h(\sigma) \sigma^{\alpha} e^{-\beta \mu_{\nu, s}^{(\mathrm{exc})}(\sigma)}}{\gamma \sum_{\nu} e^{-\beta \mu_{\nu, \mathrm{N}}^{(\mathrm{exc})}(\sigma)}+3(1-\gamma) e^{-\beta \mu_{\mathrm{I}}^{(\mathrm{exc})}(\sigma)}},
$$

where

$$
\beta \mu_{\nu, s}^{(\mathrm{exc})}(\sigma)=\sum_{\alpha=0}^{2} \frac{\partial \Phi_{\mathrm{exc}}}{\partial \rho_{\nu, s}^{(\alpha)}} \sigma^{\alpha}
$$

are the excess part of the chemical potential of species $\nu$ of width $\sigma$ in the phase $s$. Note that this is a quadratic polynomial in $\sigma$ whose coefficients are in turn functions of the moments $\rho_{\nu, s}^{(\alpha)}$ [29]. We use the notation $\rho_{\mathrm{I}}^{(\alpha)} \equiv \rho_{\nu, \mathrm{I}}^{(\alpha)}$ and $\mu_{\mathrm{I}}^{(\mathrm{exc})}(\sigma) \equiv \mu_{\nu, \mathrm{I}}^{(\mathrm{exc})}(\sigma) \forall \nu$. Thus the set of nine equations (5) (which guarantee the equality of chemical potentials of all species in both phases) are solved self-consistently for the moments $\rho_{\nu, s}^{(\alpha)}$, while the other quantity to be determined, $\rho_{0}$, is found by imposing the condition of mechanical equilibrium, i.e. the equality of pressures [see Eq. (A8)] in both phases:

$$
P_{\mathrm{I}}\left(\rho_{0},\left\{\rho_{\mathrm{I}}^{(\alpha)}\right\}\right)=P_{\mathrm{N}}\left(\rho_{0},\left\{\rho_{\nu, \mathrm{N}}^{(\alpha)}\right\}\right)
$$

The fluid pressure can be found from (A8). The cloudI-shadow-N coexistence, corresponding to a situation where the system volume is occupied by the I phase except for a coexisting, infinitesimal amount of the $\mathrm{N}$ phase, is obtained by taking $\gamma=0$ in the expressions above. The case of the shadow-I-cloud-N coexistence, which corresponds to the opposite case (i.e. the $\mathrm{N}$ phase occupying the whole system volume but in coexistence with an infinitesimal amount of the I phase), is obtained by taking $\gamma=1$.

\section{B. Length distribution function}

In the present study we choose a probability distribution function $h(\sigma)$ which is, in the general case, bimodal:

$$
h(\sigma)=\frac{x}{\sigma_{1}} h_{0}\left(\frac{\sigma}{\sigma_{1}}\right)+\frac{(1-x)}{\sigma_{2}} h_{0}\left(\frac{\sigma}{\sigma_{2}}\right) .
$$

Here $x$ is the molar fraction when the fluid is strictly a binary mixture; otherwise $x$ can be regarded as a parameter which controls the relative heights of the two maxima, located at $\sigma_{1}$ and $\sigma_{2}$. The function $h_{0}(u)$ is selected to be

$$
h_{0}(u)=C u^{\nu} e^{-\Lambda u^{p}},
$$


where the constants $C$ and $\Lambda$ are calculated from the normalization conditions $\int_{0}^{\infty} d u h_{0}(u)=\int_{0}^{\infty} d u u h_{0}(u)=$ 1. Thus we find

$$
\Lambda^{1 / p}=\frac{\Gamma[(\nu+2) / p]}{\Gamma[(\nu+1) / p]}, \quad C=\frac{p \Lambda^{(\nu+1) / p}}{\Gamma[(\nu+1) / p]},
$$

with $\Gamma(x)$ the Gamma function. For fixed $p$ the parameter $\nu$ controls the polydispersity, while $p$ controls the decay of the distribution at large $\sigma$ (note that for $p=1$ a Schultz distribution is obtained). All these definitions guarantee the normalization $\int_{0}^{\infty} d \sigma h(\sigma)=1$. For the first moment we find $\int_{0}^{\infty} d \sigma \sigma h(\sigma)=x \sigma_{1}+(1-x) \sigma_{2}$. The one-component limit is recovered by setting $x=1$ and consequently $\langle\sigma\rangle=\sigma_{1}$. Defining the polydisperse coefficient for $h_{0}(u)$ as the mean-square deviation, $\Delta_{0}=$ $\sqrt{\left\langle u^{2}\right\rangle_{0} /\langle u\rangle_{0}^{2}-1}$, where $\left\langle u^{\alpha}\right\rangle_{0} \equiv \int_{0}^{\infty} d u u^{\alpha} h_{0}(u)$, we find that the polydispersity coefficient for the bimodal distribution function $h(\sigma)$, i.e. $\Delta=\sqrt{\left\langle\sigma^{2}\right\rangle /\langle\sigma\rangle^{2}-1}$ [with $\left.\left\langle\sigma^{\alpha}\right\rangle \equiv \int_{0}^{\infty} d \sigma \sigma^{\alpha} h(\sigma)\right]$ results in

$$
\Delta=\frac{1}{\bar{\sigma}} \sqrt{\overline{\sigma^{2}} \Delta_{0}^{2}+x(1-x)\left(\sigma_{2}-\sigma_{1}\right)^{2}},
$$

where we have defined $\overline{\sigma^{\alpha}} \equiv x \sigma_{1}^{\alpha}+(1-x) \sigma_{2}^{\alpha}$. As $\overline{\sigma^{2}}>\bar{\sigma}^{2}$ for $x \neq 0$ or 1 , we find $\Delta>\Delta_{0}$. For a fixed parameter $r \equiv$ $\sigma_{2} / \sigma_{1}>1$, the polydispersity coefficient as a function of $x, \Delta(x)$, has a maximum at $x^{*}=\frac{r}{r+1}$ with value

$$
\Delta\left(x^{*}\right)=\frac{r+1}{2 \sqrt{r}} \sqrt{\Delta_{0}^{2}+\left(\frac{r-1}{r+1}\right)^{2}} .
$$

For example, setting $\Delta_{0}=0.3$, and for $r=1.5,2,2.5$ and 3 , we find $\Delta\left(x^{*}\right)=0.368,0.476,0.579$ and 0.673 respectively; we then see that the bimodality dramatically increases the effective polydispersity $\Delta$ of the mixture.

To present the results in the following sections we use the packing or volume fraction $\eta_{s}(\gamma)$ with $s=\mathrm{I}$, N, which is a function of $\gamma$, and is defined through the zeroth moment of the distribution function as $\eta_{s}(\gamma) \equiv \rho_{s}^{(0)}(\gamma) \sigma_{1}^{2} L$. Specifically we will use for the presentation of results the values $\eta_{\mathrm{I}} \equiv \eta_{\mathrm{I}}(0)=\rho_{0}(0) \sigma_{1}^{2} L$ and $\eta_{\mathrm{N}} \equiv \eta_{\mathrm{N}}(1)=$ $\rho_{0}(1) \sigma_{1}^{2} L$, i.e. the cloud-I and cloud-N packing fractions. Also we use the total packing fraction of the polydisperse mixture $\eta(\gamma) \equiv \gamma \eta_{\mathrm{N}}(\gamma)+(1-\gamma) \eta_{\mathrm{I}}(\gamma)=\rho_{0}(\gamma) \sigma_{1}^{2} L$, the latter equality being a consequence of the lever rule. Finally we will use the length distribution functions corresponding to coexisting phases defined as $h^{(s)}(\sigma) \equiv$ $\rho_{0}^{-1} \sum_{\nu} \rho_{\nu, s}(\sigma),(s=\mathrm{I}, \mathrm{N})$. Again, using the lever rule, we have $\gamma h^{(\mathrm{N})}(\sigma)+(1-\gamma) h^{(\mathrm{I})}(\sigma)=h(\sigma)$.

\section{Polydispersity in particle shape}

In most colloidal suspensions of anisotropic particles, polydispersity exists not only in particle size but also in particle shape. As the inclusion in the theory of both types of polydispersities constitutes a highly nontrivial task, the usual procedure is to map the real particles onto effective particles of fixed shape but with an effective polydispersity in their sizes. In the following we describe how the effective polydispersity can be calculated in our particular system in which the main quantity that governs phase behavior is the particle area of the transverse section (we note again that the thickness $L$ is constant).

We consider a system made of a collection of particles of fixed thickness $L$ and different cross sections. To be more precise, we suppose the latter to have the form of regular polygons inscribed in circumferences of different diameters $2 R$ and also with different number of edge-lengths $n$. Thus our system is polydisperse in the variables $R$ and $n$, the former controlling the size polydispersity, while the latter controls the particle shape. The cross-sectional area of these particles can be calculated as $A_{n}(R)=\frac{n R^{2}}{2} \sin \left(\frac{2 \pi}{n}\right)$. Suppose the polydisperse coefficient [mean square deviation of the probability distribution function $h(R)]$ is $\Delta$, and we define the probability to find a polygon with $n$ edge-lengths as $p_{n}$. Now we map our system onto an effective one, monodisperse in the number $n_{0}$ of edge-lengths, but with an effective polydispersity $\Delta_{\text {eff }}$. We define the mapping as

$$
\left\langle A_{n_{0}}\right\rangle_{h_{\mathrm{eff}}}=\sum_{n} p_{n}\left\langle A_{n}\right\rangle_{h}
$$

where

$$
\left\langle A_{n}\right\rangle_{h}=\int d R h(R) A_{n}(R)=\frac{n\langle R\rangle_{h}^{2}\left(1+\Delta^{2}\right)}{2} \sin \left(\frac{2 \pi}{n}\right) .
$$

We take $\langle R\rangle_{h}=\langle R\rangle_{h_{\text {eff }}}$, i.e. the mean radii that follow from the distribution functions $h(R)$ and $h_{\mathrm{eff}}(R)$ are exactly the same. Defining the coefficient

$$
q=\frac{\sum_{n} p_{n} n \sin (2 \pi / n)}{n_{0} \sin \left(2 \pi / n_{0}\right)}
$$

we find that the effective polydispersity coefficient can be found as

$$
\Delta_{\mathrm{eff}}=\sqrt{q\left(1+\Delta^{2}\right)-1}
$$

We have used the following expression for the probability $p_{n}$ :

$$
p_{n}=\frac{(n-4+k) !}{(n-4) ! k !} p^{n-4}(1-p)^{k+1}, \quad n \geq 4,
$$

with the triangular shape excluded, which is usually the case in experiments. These probabilities fulfill the normalization condition $\sum_{n=4}^{\infty} p_{n}=1$. Once we fix the mean number of edge-lengths $\langle n\rangle$, the value of $p$, a function of 


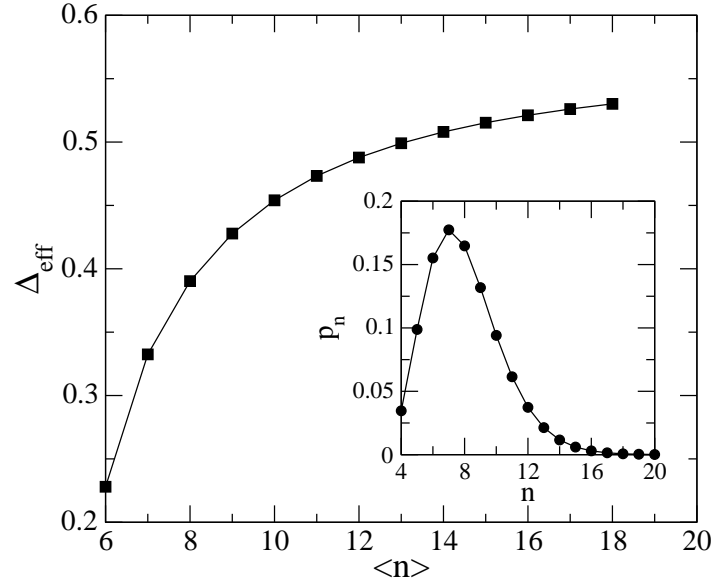

FIG. 1: Effective polydispersity $\Delta_{\text {eff }}$ as a function of the mean number of edge-lengths $\langle n\rangle$ for $n_{0}=6, \Delta_{0}=0.3$ and $k=9$. Inset: probability $p_{n}$ as a function of $n$ for $k=9$ and $\langle n\rangle=8$.

$\langle n\rangle$ and $k$, has the form $p=\frac{\langle n\rangle-4}{\langle n\rangle+k-3}$. The polydispersity in the number of edge-lengths can be quantified through the coefficient

$$
\Delta_{n} \equiv \sqrt{\frac{\left\langle n^{2}\right\rangle}{\langle n\rangle^{2}}-1}=\sqrt{\frac{1}{\langle n\rangle-4}+\frac{1}{k+1}} .
$$

Thus the number $k$ controls the polydispersity. In Fig. 1 we plot the effective polydispersity coefficient $\Delta_{\text {eff }}$ as a function of $\langle n\rangle$ for the case $n_{0}=6, \Delta_{0}=0.3$ and $k=9$. As can be seen from the figure, $\Delta_{\text {eff }}$ is a monotonically increasing function of $\langle n\rangle$ and it can reach values above 0.5. A particular example of $p_{n}$ (for $k=9$ and $\langle n\rangle=8$ ) is plotted in the inset.

\section{RESULTS}

We have carried out coexistence calculations following the procedure described in Sec. ЩA . First we consider a polydisperse mixture with unimodal distribution function $(x=1)$ and Gaussian tail $(q=2)$, and vary the polydisperse coefficient in the range $0 \leq \Delta_{0} \leq 0.75$. The results are plotted in Fig. 2 2 in the $\eta_{s}-\Delta_{0}$ phase diagram for four different values of the aspect ratio: $\kappa=L / \sigma_{1}=0.2$, $0.1,0.02$, and 0.01 .

The main conclusions we can draw from these results can be summarized as follows: (i) the two-phase region is broadened as polydispersity is increased (in agreement with other theoretical and experimental results); (ii) this effect is enhanced as $\kappa$ is lowered, and (iii) the behaviour of $\eta_{\mathrm{N}}$ as a function of $\Delta_{0}$ changes with $\kappa$ : for relatively high values of $\kappa$ it is a decreasing function, whereas for low enough $\kappa$ it becomes an increasing function for large $\Delta_{0}$

In a recent experiment [30], suspensions of polydisperse platelets were prepared from exfoliation of pris-
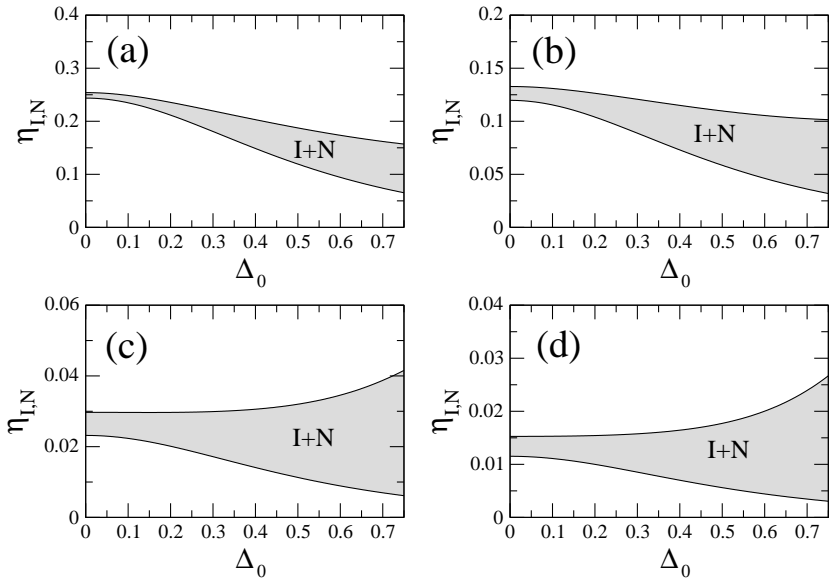

FIG. 2: The cloud-I and cloud-N coexisting packing fractions $\eta_{\mathrm{I}}$ and $\eta_{\mathrm{N}}$ as a function of polydispersity $\Delta_{0}$ for values of $\kappa$ equal to: (a) 0.2 , (b) 0.1 , (c) 0.02 and (d) 0.01 .

tine $\alpha$-ZrP crystals using TBA molecules. The resulting platelets were found to be polydisperse in diameter and also in shape (although most particles have approximately hexagonal geometry), with a constant thickness equal to $26.8 \AA$. Different aqueous suspensions were prepared with particles of mean aspect ratio [as measured by dynamic light scattering (DLS)] ranging from 0.001 to 0.01 . The polydispersity coefficients of the suspensions were estimated using Dynamic Light Scattering (DLS), and all of them were found to be in the range 18\%-39\%, with most samples having a value of about $30 \%$. Samples were divided into three different sets: A, B and C. Samples corresponding to set $\mathrm{B}$ were obtained from nematic fractionation of an original suspension followed by dilution and, consequently, this set has the lowest polydispersity coefficients. The other two sets, A and C, result from the original synthesis and exfoliation of the pristine crystals. All platelets have negative surface charges which are partially neutralized by the positive charges of the TBA molecules, thus creating effective dipoles. Nonneutralized charges and dipoles cause the pair-interaction between two platelets to be long-ranged and repulsive.

\section{A. Unimodal length distribution}

With the aim of modeling the fluid, we mapped a collection of repulsive, shape- and diameter-polydisperse platelets onto effective polydisperse board-like particles interacting through excluded volume. To properly take into account the effect of long-ranged repulsive interactions, the effective thickness of particles has to be larger than the thickness of the actual colloidal platelets, and the effective aspect ratios $\kappa_{\text {eff }} \equiv \frac{L_{\text {eff }}}{\langle\sigma\rangle}=\kappa \frac{L_{\text {eff }}}{L}$ are obtained from the real $\kappa$ by scaling by a factor $f \equiv \frac{L_{\text {eff }}}{L}>1$. As polydispersity increases, $f$ should also increase due to 

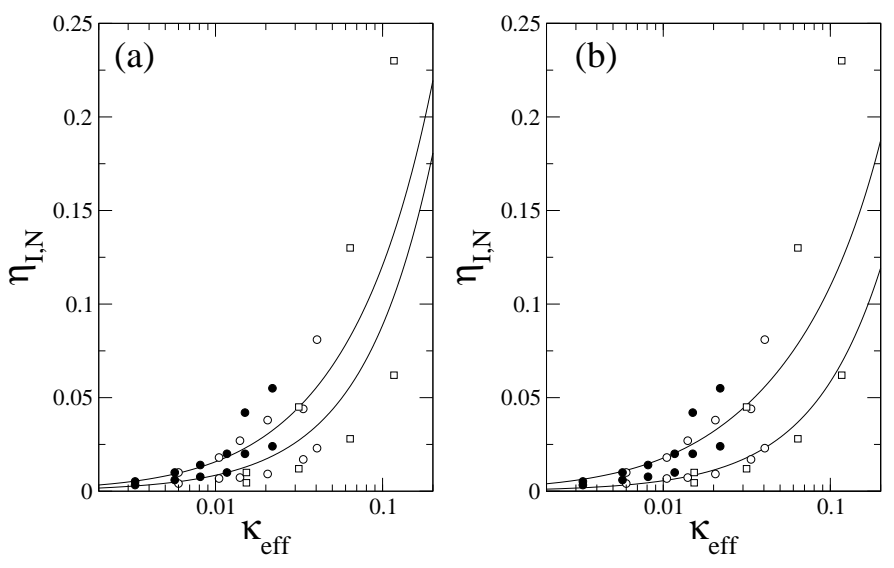

FIG. 3: The cloud coexisting packing fractions $\eta_{\mathrm{I}, \mathrm{N}}$ as a function of the aspect ratio $\kappa_{\text {eff }}$ for polydispersities fixed to (a) $\Delta_{0}=0.3$, and (b) 0.5. Open circles, filled circles and open squares correspond to the experimental results in Ref. 30. for sets $\mathrm{A}, \mathrm{B}$ and $\mathrm{C}$ (adequately rescaled with factors $f=5$, 3 and 9 , respectively).

the presence of platelets with large surface area which, as discussed above, should repel each other more strongly.

This effect is in fact obtained with the model, as shown in Fig. 3, where the cloud packing fractions $\eta_{\mathrm{I}, \mathrm{N}}$ are plotted as a function of $\kappa_{\text {eff }}$ for two values of polydispersity, $\Delta_{0}=0.3$ in (a) and $\Delta_{0}=0.5$ in (b), and using a unimodal length distribution $h(\sigma)$ with $q=2$. Also included in the figures are the experimental results from [30] with $f$ set to 5,3 and 9 for samples $\mathrm{A}, \mathrm{B}$ and $\mathrm{C}$, respectively. These values were chosen to ensure a proper agreement between theory and experiment (note that no least-square optimization was attempted). Samples in set B are less polydisperse, and consequently $f$ is smaller. As can be seen from the figure, these samples are relatively well described by our model using $\Delta_{0}=0.3$ (as in the actual samples), except for those experimental points with the two higher values of $\kappa_{\text {eff }}$. However, for samples in set A, better agreement is obtained with $\Delta_{0}=0.5$. Note that polydispersity in the experimental samples is in diameter and also in shape which, as discussed in Sec. IIC, demands that the effective polydispersity of a single-shaped model be higher. Finally, samples in set $\mathrm{C}$, which are characterized by huge coexistence gaps, are not well described by unimodal length distributions. The multimodality of the distribution is probably behind this behaviour (see following section).

Fig. 4 shows the percentage of total volume $\gamma$ occupied by the $\mathrm{N}$ phase as a function of the total packing fraction $\eta$ of the mixture for those values of $\kappa_{\text {eff }}$ corresponding to the sets A [Fig. 4(a)] and B [Fig. 4(b)]. In the former case solid lines are results from calculations with a unimodal distribution using $q=2$ and $\Delta_{0}=0.5$, while in the latter case $\Delta_{0}=0.3$. It is clear that when $\Delta_{0}=0.3$ the function $\gamma(\eta)$ is practically a linear function [see Fig. 4 (b)], while for $\Delta_{0}=0.5$ some nonlinearity is already apparent, a trend which is more pronounced in experi-
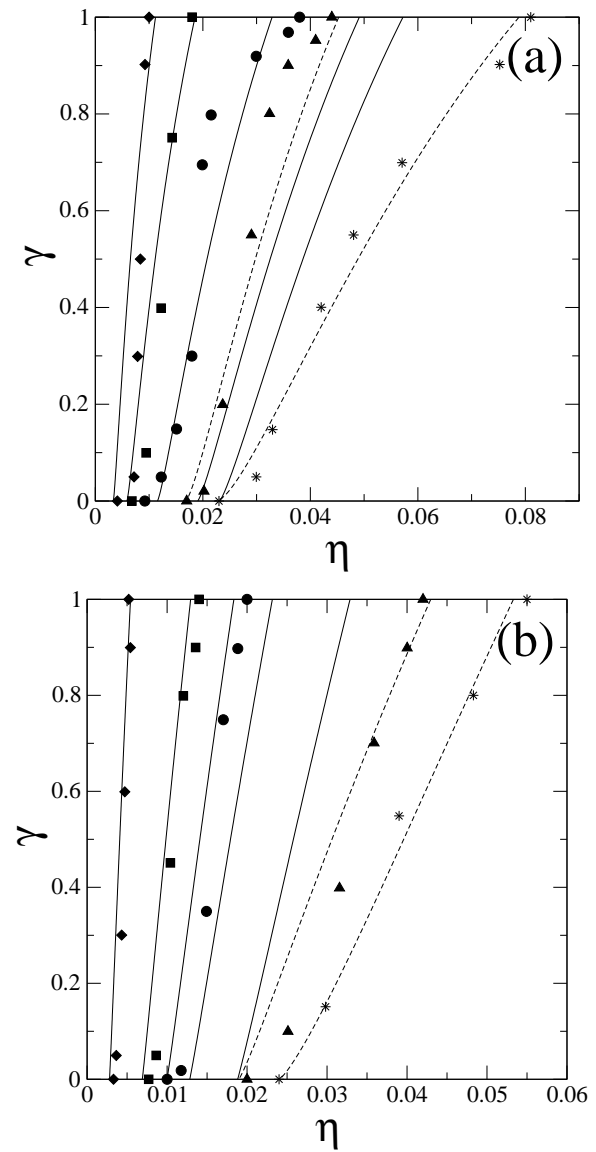

FIG. 4: Percentage of total volume $\gamma$ occupied by $\mathrm{N}$ phase as a function of total packing fraction $\eta$ for values of $\kappa_{\text {eff }}$ corresponding to the experimental sets A, panel (a), and B, panel (b). Rhombi, squares, circles, triangles and stars are used to show the experimental results from [30] as $\kappa_{\text {eff }}$ is increased. Solid lines correspond to the theoretical results obtained from a unimodal length distribution with $q=2$ and (a) $\Delta_{0}=0.5$, (b) $\Delta_{0}=0.3$. Theoretical results using a bimodal distribution are represented by dashed curves. Values of parameters that better describe experimental data represented by stars and triangles are as follows. Panel (a): $\Delta_{0}=0.5, q=2$, $\sigma_{1} / L_{\text {eff }}=10, \sigma_{2} / L_{\text {eff }}=17.5$ and $x=0.7$ for stars, and $\sigma_{1} / L_{\text {eff }}=20, \sigma_{2} / L_{\text {eff }}=26$ and $x=0.45$ for triangles. Panel (b): $\Delta_{0}=0.3, q=2, \sigma_{1} / L_{\mathrm{eff}}=20, \sigma_{2} / L_{\mathrm{eff}}=32$ and $x=0.78$ for stars, and $\sigma_{1} / L_{\text {eff }}=20, \sigma_{2} / L_{\text {eff }}=32.6$ and $x=0.65$ for triangles.

ment. Coexistence gaps from theory and experiment are similar, except for the highest value of $\kappa_{\text {eff }}$ in set A [stars in Fig. 4 (a)], and for the two higher values of $\kappa_{\text {eff }}$ in set $\mathrm{B}$ [triangles and stars in (b)]. A possible reason for these deviations will also be explained in the following section.

\section{B. Bimodal distribution}

In this section we demonstrate that the multimodality of the length distribution function can explain both the 
existence of a huge I-N coexistence gap, and the strong nonlinearity of $\gamma(\eta)$. These are two of the main features present in the experiments of [30] for those samples with higher values of $\kappa_{\text {eff }}$, i.e. samples in sets $\mathrm{A}$ and B. For set $\mathrm{C}$ we show below that the bimodality is crucial to adequately describe experimental results. The origin of this behavior is the coupling between the fractionation effect, typical of polydisperse mixtures, and the demixing phenomenon that occurs in multicomponent mixtures of particles with sufficiently different lengths. In binary mixtures with very asymmetric species, entropy forces the system to segregate into two phases of very different composition, and consequently the coexistence density gap is very large compared to that in one component systems or in polydisperse mixtures with unimodal size distributions.

Dashed lines in Fig. 4 are the theoretical predictions for $\gamma(\eta)$ in the case of the experimental sets A and B [triangles and stars in panels (a) and (b), respectively]. Calculations were based on a bimodal parent distribution function $h(\sigma)$, as described in Sec. IIB, and the corresponding functions for the different cases are shown in Figs. 5 (a) and (c) (solid lines). Values for the parameters in $h(\sigma)$ were chosen so as to optimise agreement with the experimental data [stars in Figs. 4 (a) and (b)]. As can be seen from Figs. [5 the parent distribution function seems to be unimodal in both cases, A and B, even though compositions close to $50 \%$ were chosen $(x=0.70$ and $x=0.78$, respectively). The high polydispersity $\left(\Delta_{0}=0.5\right.$ and 0.3 , respectively) creates a large overlap region between the two distribution functions centered at $\sigma_{1}$ and $\sigma_{2}$ [see Eqn. (8)], which results in the absence of a second maximum near $\sigma_{2}$.

The combined effect of fractionation and demixing can also be observed in Fig. 5 from the shape of the distribution functions for the shadow-N phase [dashed curves in panels (a) and (c)]. Note that the fraction of platelets with lengths $\sigma \sim \sigma_{1}$ is much lower than that in the coexisting (parent) I phase, but the fraction for lengths $\sigma \geq \sigma_{2}$ increases dramatically. As a result, there appears a shoulder in the distribution function, and its decay for large $\sigma$ is much slower. The opposite effect occurs for the shadow-I distribution functions [solid lines in panels (b) and (d)]: now the I phase is rich in platelets with $\sigma \sim \sigma_{1}$, while the distribution function decays much faster and the polydisperse mixture is poor in large platelets.

As mentioned above, the most dramatic disagreement between the theoretical calculations based on unimodal distributions and the experimental results corresponds to samples in set C (see Fig. 3). One possible reason for this disagreement is the decay rate of the parent distribution function, controlled by the parameter $q$. In order to check this, we have implemented our calculations using a truncated unimodal distribution with $q=1$ (Schultz distribution), $\Delta_{0}=0.5$ and the same values of $\kappa_{\text {eff }}$ as in Fig. 3. The results, represented by means of dashed lines in Fig. 6(a), give a broad coexistence gap (note that for vanishingly small values of $\gamma$, the total packing fraction
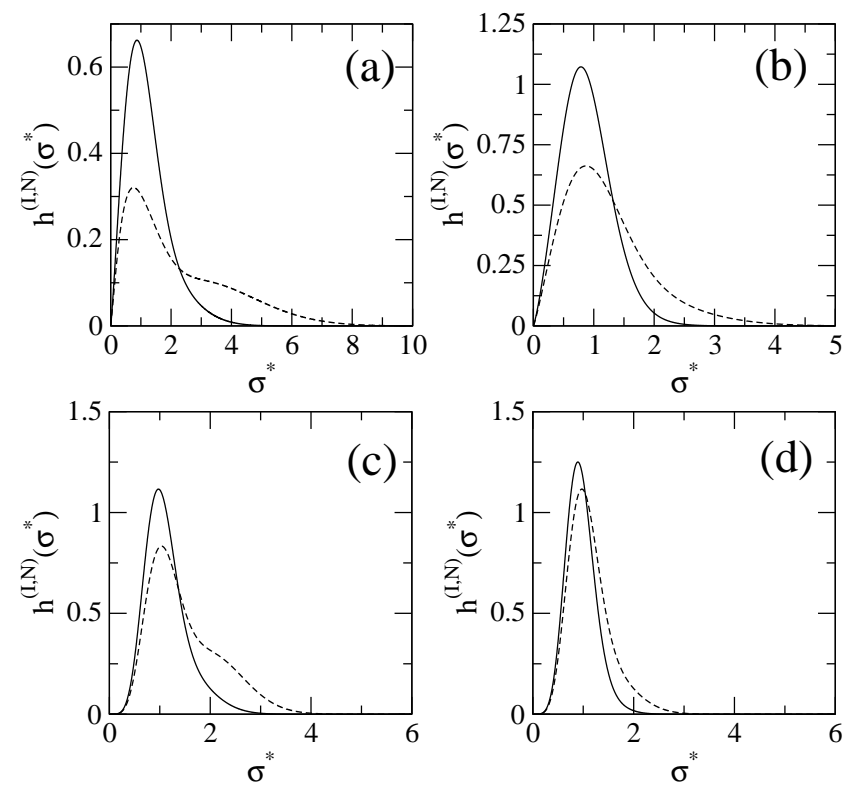

FIG. 5: Length distribution functions $h^{(\mathrm{I}, \mathrm{N})}\left(\sigma^{*}\right)$, as a function of reduced length $\sigma^{*}=\sigma / \sigma_{1}$, of coexisting I (solid curves) and $\mathrm{N}$ (dashed curves) phases. Panels (a) and (c) correspond to cloud-I-shadow- $\mathrm{N}$ coexistence (i.e. $\gamma=0$ ), while (b) and (d) refer to cloud-N-shadow-I coexistence $(\gamma=1)$. The parent distribution function, which coincides with that of the cloudI (for $\gamma=0$ ) or cloud-N (for $\gamma=1$ ) cases, is bimodal with the following parameters: panels (a) and (b), $\Delta_{0}=0.5, q=2$, $\sigma_{1} / L_{\text {eff }}=10, \sigma_{2} / L_{\text {eff }}=17.5$ and $x=0.70 ;$ panels (c) and (d), $\Delta_{0}=0.3, q=2, \sigma_{1} / L_{\text {eff }}=20, \sigma_{2} / L_{\text {eff }}=32$ and $x=0.78$.

$\eta$ rapidly increases from $\eta_{\mathrm{I}}$, which is shown by stars), but they fail to reproduce the experiments. Also, the nonlinearities of the curves $\gamma(\eta)$ are not correctly described.

In the same figure, the theoretical results using bimodal distribution functions with $q=2$ and $\Delta_{0}=0.5$ are also plotted. The parameters $\sigma_{i} / L_{\text {eff }}$ and $x$ were chosen to guarantee a reasonable agreement between theory and experiment so that now both the coexistence gaps and also the nonlinear behavior of $\gamma(\eta)$ are reproduced. Fig. 6(b) shows the bimodal parent and shadow-I,N coexisting length distributions (corresponding to those parameters which better describe the experimental points of Fig. 6(a) represented by circles). Note that the bimodal parent distribution function looks unimodal. Also, the shadow- $\mathrm{N}$ distribution exhibits a plateau in the range of scaled lengths $7-12$, while the shadow-I is highly localized about the value 1 .

To illustrate how a strong nonlinearity in $\gamma(\eta)$ can emerge from the coupling between polydispersity and bimodality, Fig. 7(a) shows $\gamma$ as obtained from calculations using a bimodal distribution with $q=2, \Delta_{0}=0.3$, $x=0.85, \sigma_{1} / L_{\text {eff }}=20$ and different values of $\sigma_{2} / L_{\text {eff. }}$. As the latter is increased, the nonlinearity becomes stronger up to a point where there appears a well-defined loop in $\gamma(\eta)$ [see inset in Fig. [7(a)]. We should mention here that the presence of this loop is not related with a triple $\mathrm{I}-\mathrm{N}_{1}-\mathrm{N}_{2}$ or $\mathrm{I}_{1}-\mathrm{I}_{2}-\mathrm{N}$ coexistence, a fact we have proved by 

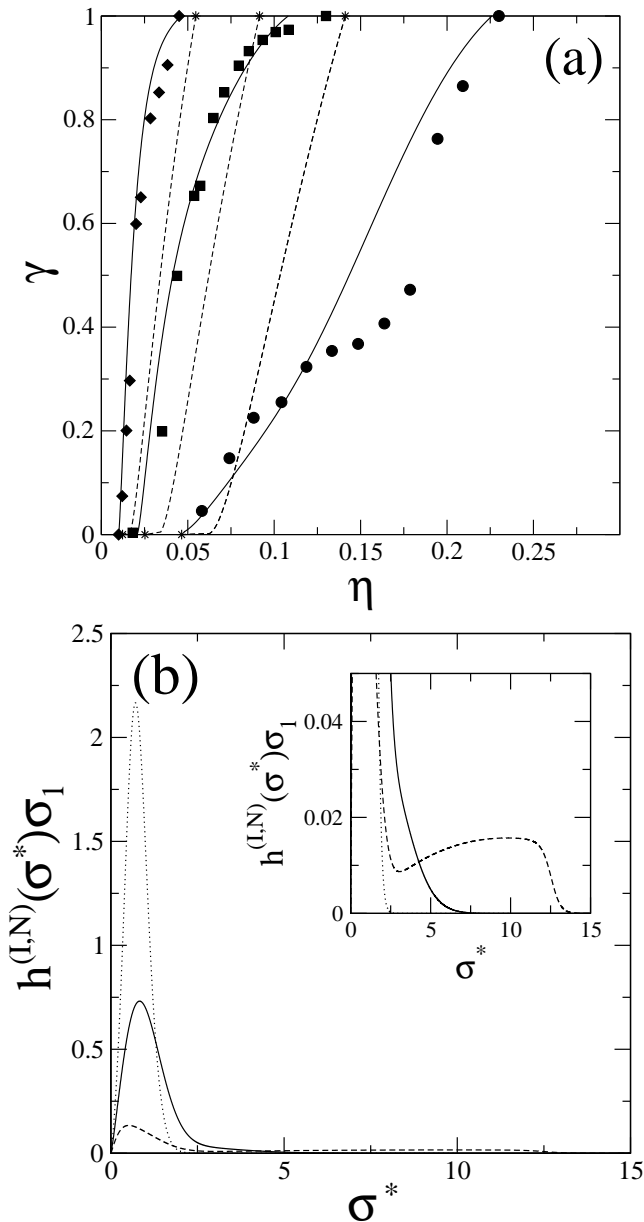

FIG. 6: (a) Percentage of total volume occupied by $\mathrm{N}$ phase as a function of total packing fraction $\eta$ for values of $\kappa_{\text {eff }}$ corresponding to the experimental samples in set $\mathrm{C}$ [30]. Rhombus, squares and circles are used to represent the experimental values for increasing $\kappa_{\text {eff }}$. Dashed lines: results for the same value of $\kappa_{\text {eff }}$ but using a truncated Schultz distribution function $(q=1)$ with $\Delta_{0}=0.5$. Stars indicate values of $\eta_{\mathrm{I}}$ and $\eta_{\mathrm{N}}$ for each $\kappa_{\text {eff }}$. Solid lines: results from a bimodal distribution functions with $q=2$ and $\Delta_{0}=0.5$; the other parameters specifying the degree of bimodality we give only for the curve that better describes the experimental data shown with circles. They are: $\sigma_{1} / L_{\text {eff }}=4, \sigma_{2} / L_{\text {eff }}=12$ and $x=0.9$. (b) Length distribution functions for the latter set of parameters. Solid, dashed and dotted lines correspond to parent, shadow$\mathrm{N}$ and shadow-I distribution functions, respectively. Zoom of (b) shows second maximum in shadow- $\mathrm{N}$ distribution function.

solving the set of equations for the moments and pressures of the different coexisting phases and checking that they always converge to solutions corresponding to I-N coexistence. Fig. 7 (b) shows the distribution functions $h^{(\mathrm{I}, \mathrm{N})}(\sigma)$ for the three different I-N coexistences occurring for $\gamma=0.2175$ [symbols in inset of panel (a)] inside the loop. Clearly, as $\eta$ is increased, the function $h^{(\mathrm{N})}(\sigma)$ becomes less peaked at $\sigma_{1}$ but more peaked about $\sigma_{2}$ and
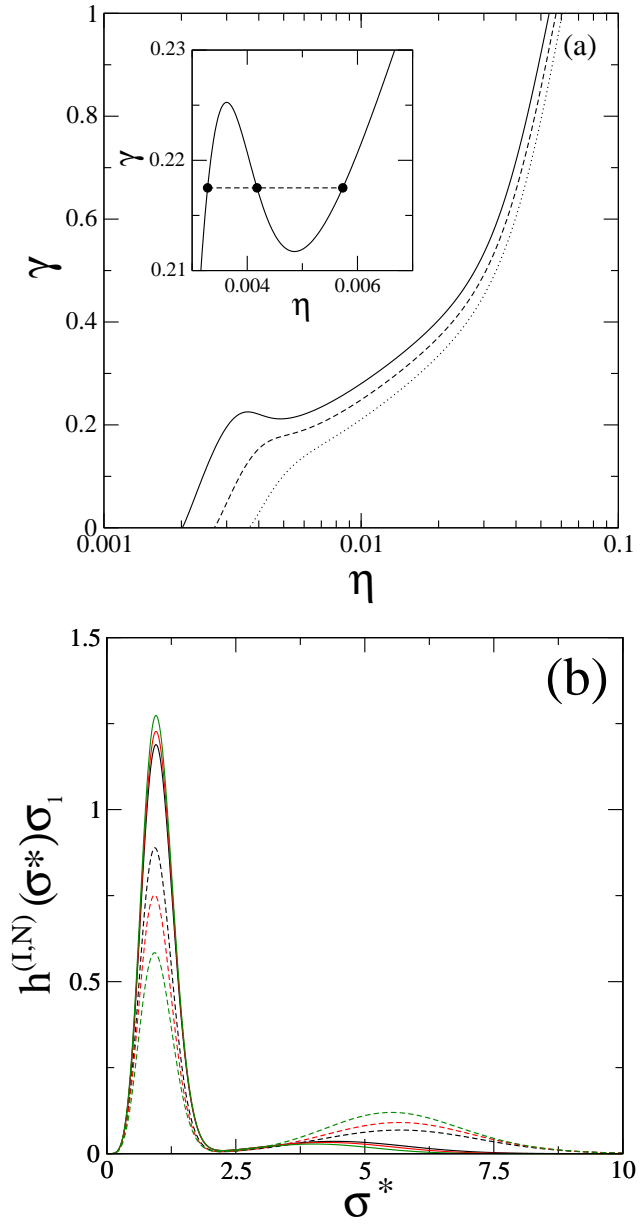

FIG. 7: (a) $\gamma$ versus $\eta$ in logarithmic scale as obtained from a bimodal distribution with $q=2, \Delta_{0}=0.3, x=0.85$, $\sigma_{1} / L_{\text {eff }}=20$ and $\sigma_{2} / L_{\text {eff }}=80$ (dotted curve), 90 (dashed curve) and 100 (solid curve). Zoom in (a) is a detail of the latter case showing the loop in the function $\gamma(\eta)$. (b) Three pairs of coexisting distribution functions $h^{(\mathrm{I}, \mathrm{N})}\left(\sigma^{*}\right)$ corresponding to the points shown in (a) for $\gamma=0.2175$. Solid and dashed lines represent I and $\mathrm{N}$ phases, respectively, while different colors represent different values of $\eta$ (black, red and green in the order of increasing $\eta$ ).

with a slower decay for large $\sigma$, while the function $h^{(\mathrm{I})}(\sigma)$ remains practically the same. The presence of this loop is clearly related with the density inversion phenomenon. As can be seen in Fig. 7 (b), the moments of the I and $\mathrm{N}$ coexisting distribution functions fulfill the inequalities $\rho_{\mathrm{N}}^{(2)}>\rho_{\mathrm{I}}^{(2)}$ and $\rho_{\mathrm{N}}^{(0)}<\rho_{\mathrm{I}}^{(0)}$ [due to the defect (excess) of platelets of width close to $\sigma_{1}\left(\sigma_{2}\right)$ in the coexisting $\mathrm{N}$ phase with respect to the I phase]. As we have defined the total packing fraction $\eta$ as being proportional to the zeroth moment, and this moment is lower for the $\mathrm{N}$ phase, the density inversion is produced.

To better understand this behavior, we resort to the lever rule (1). Dividing the whole equation by $\rho_{0}$ and 
integrating over $\sigma$, we find

$$
1=\gamma\left(\rho_{0}\right) A_{\mathrm{N}}\left(\rho_{0}\right)+\left[1-\gamma\left(\rho_{0}\right)\right] A_{\mathrm{I}}\left(\rho_{0}\right),
$$

where we have defined $A_{\mathrm{s}} \equiv \int d \sigma h^{(s)}(\sigma)$ as the total area under the curve $h^{(s)}(\sigma)(s=\mathrm{I}, \mathrm{N})$. Both these areas and $\gamma$ are functions of the parent number density $\rho_{0}$. From (19) we find that the derivative of $\gamma$ with respect to $\rho_{0}$ is

$$
\gamma^{\prime}=\frac{A_{\mathrm{N}}^{\prime}\left(A_{\mathrm{I}}-1\right)+A_{\mathrm{I}}^{\prime}\left(1-A_{\mathrm{N}}\right)}{\left(A_{\mathrm{N}}-A_{\mathrm{I}}\right)^{2}}
$$

In those polydisperse fluids where the parent distribution function is strictly unimodal, density inversion does not occur and we have that $A_{\mathrm{I}} \leq 1$ and $A_{\mathrm{N}} \geq 1$. Taking the latter inequalities into account and the fact that $\rho_{0}$ goes from $\rho_{0}^{(\mathrm{I})}$ to $\rho_{0}^{(\mathrm{N})}$ (the values of the parent number densities corresponding to the cloud-I-shadow-N coexistence, with $A_{\text {cloud-I }}=1$, and the cloud-N-shadow I coexistence, with $A_{\text {cloud-N }}=1$, respectively), we have most likely that $A_{\mathrm{I}}^{\prime}<0$ and $A_{\mathrm{N}}^{\prime}<0$. Thus we find from Eq. (20) that $\gamma^{\prime}>0$ in the whole interval $\left[\rho_{0}^{(\mathrm{I})}, \rho_{0}^{(\mathrm{N})}\right]$. When the parent distribution function is bimodal, density inversion could occur. For the latter situation we have the opposite scenario: $A_{\mathrm{I}} \geq 1$ and $A_{\mathrm{N}} \leq 1$. Thus we could conclude that $A_{\mathrm{I}}^{\prime}>0$ and $A_{\mathrm{N}}^{\prime}>0$ and then we obtain again $\gamma^{\prime}>0$ [see Eq. (20)] in the whole range of $\rho_{0}$. However for strong bimodality [when the two peaks of $h(\sigma)$ are well visible as shown in Fig. 7 (b)], the sign of $A_{\mathrm{N}}^{\prime}$ could change from positive to negative giving rise, for certain values of $\rho_{0}$, to $\gamma^{\prime}<0$. To elucidate the conditions necessary for having a negative sign of $\gamma^{\prime}$, we resort again to Eq. (20). From the latter it is easy to show that, if $\gamma^{\prime}<0$, we obtain the condition

$$
\frac{d}{d \rho_{0}} \ln \left|1-A_{\mathrm{N}}\right|>\frac{d}{d \rho_{0}} \ln \left|1-A_{\mathrm{I}}\right| .
$$

In Fig. 8 we plot the functions $S\left(\rho_{0}\right)=\frac{d}{d \rho_{0}} \ln \left|1-A_{s}\right|$ when $h(\sigma)$ is unimodal (a) and bimodal (b) (that corresponding to the results shown in Fig. 7). We can see that while the condition (21) is not fulfilled for any $\rho_{0}$ for the unimodal $h(\sigma)$, there exists, with a bimodal $h(\sigma)$, a range of $\rho_{0}$ (shaded in the figure with grey color) for which this condition is fulfilled. It is easy to show, using Eq. (19), that the condition (21) is equivalent to the following inequality

$$
\gamma\left|A_{\mathrm{N}}^{\prime}\right|>(1-\gamma) A_{\mathrm{I}}^{\prime}
$$

Thus we conclude that $\gamma^{\prime}<0$ when the rate of change in the area under the curve $h^{(N)}(\sigma)$ weighted with the factor $\gamma$ is greater than the corresponding rate of change of area of $h^{(I)}(\sigma)$ weighted with $1-\gamma$. We can see from Fig. 7 that the distribution functions $h^{(N)}(\sigma)$ corresponding to the three values of $\rho_{0}$ shown $\left(\rho_{0}^{(1)}<\rho_{0}^{(2)}<\rho_{0}^{(3)}\right)$ are dramatically different. These values are well inside the range of $\rho_{0}$ where $A_{\mathrm{N}}^{\prime}<0$ [see Fig. 8 (b)]. While the first peak
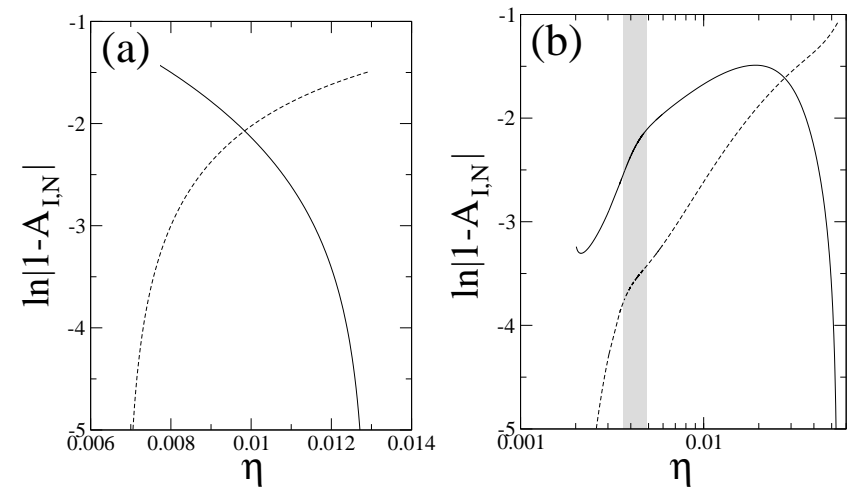

FIG. 8: $\frac{d}{d \rho_{0}} \ln \left|1-A_{\mathrm{I}, \mathrm{N}}\right|$ (dashed and solid lines correspond to I and $\mathrm{N}$ phases, respectively) as a function of $\eta=\rho_{0} \sigma_{1}^{2} L$ for $h(\sigma)$ taken as unimodal (a) and bimodal (b), the latter corresponding to the results shown in Fig. 7] The shaded region in (b) shows the interval of $\eta$ where the condition (21) is fulfilled.

centered at $\sigma \sim \sigma_{1}$ decreases, the second peak centered at $\sigma \sim \sigma_{2}$ increases. But these changes do not compensate each other, resulting in the net lowering of the total area under the curve as $\rho_{0}$ increases. We can also see that $h^{(I)}(\sigma)$ practically remains the same for these three values of $\rho_{0}$. A small increment of the first peak with $\rho_{0}$ makes $A_{\mathrm{I}}^{\prime}>0$. Finally, for $\rho_{0}=\rho_{0}^{(2)}$ the condition (22) is fulfilled and we have at this point that $\gamma^{\prime}<0$.

To end this section, we show how the behaviour of the cloud coexisting packing fractions $\eta_{\mathrm{I}, \mathrm{N}}$ as a function of $\kappa_{\mathrm{eff}} \equiv L_{\text {eff }} / \sigma_{1}$ changes with the presence of bimodality. These packing fractions are shown in Fig. 9(a) as a function of $\kappa_{\text {eff }}$; results from unimodal and bimodal distribution functions are represented by dashed and solid curves, respectively. A measure of how this function behaves is the parameter $\tau \equiv \frac{d \ln \eta_{\mathrm{I}, \mathrm{N}}}{d \ln \kappa_{\mathrm{eff}}}$. Note that if $\eta_{\mathrm{I}, \mathrm{N}} \sim a \kappa_{\mathrm{eff}}^{\alpha}$ for small $\kappa_{\text {eff }}$, we obtain $\tau \sim \alpha$, i.e. $\tau$ is a measure of the local power-law dependence of $\eta_{\mathrm{I}, \mathrm{N}}$ as a function of $\kappa_{\mathrm{eff}}$. In the limit $\kappa_{\text {eff }} \sim 0$, it can be shown that $\alpha \sim 1$ for unimodal size distributions and for both $\mathrm{I}$ and $\mathrm{N}$ curves, with the I curve having $\tau \gtrsim 1$ and the $\mathrm{N}$ curve $\tau \lesssim 1$ (the latter deviating much more from unity) [see Fig. 9(b)]. As polydispersity increases, this behavior is reached for lower values of $\kappa_{\text {eff }}$. The parameter $\tau$ as a function of $\kappa_{\text {eff }}$ is plotted in panel (b). From this figure we can see that the bimodality dramatically increases the value of $\tau$ corresponding to the cloud-I coexistence curve, while for the cloud-N coexistence curve the effect is the opposite for small enough $\kappa$, but to a lesser extent. Therefore, some caution should be exercised in extracting the powerlaw dependence of $\eta_{\mathrm{I}, \mathrm{N}}$ with $\kappa_{\text {eff }}$ by simply measuring the slope for small values of the aspect ratio. 

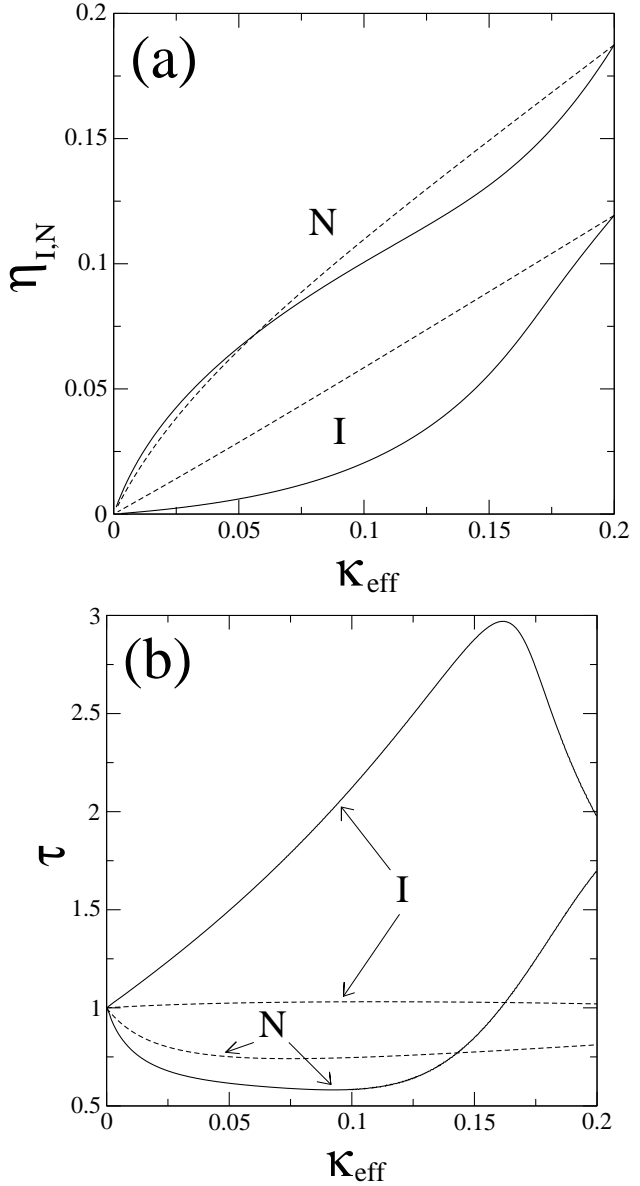

FIG. 9: (a) Cloud I and N packing fractions $\eta_{\mathrm{I}, \mathrm{N}}$ as a function of $\kappa_{\text {eff }}=L_{\text {eff }} / \sigma_{1}$ for unimodal (dashed curve) and bimodal (solid curve) distribution functions. Parameter values are set as $q=2$ and $\Delta_{0}=0.5$ for both distributions, while for the bimodal distribution $x=0.85$ and $\sigma_{2} / \sigma_{1}=a+b \kappa_{\text {eff }}$, with $a$ and $b$ chosen such that $\sigma_{2} / \sigma_{1}=1$ for $\kappa_{\text {eff }}=0.2$ and $\sigma_{2} / \sigma_{1}=3$ for $\kappa_{\text {eff }}=0.01$. (b) $\tau$ parameter, calculated from $\eta_{\mathrm{I}, \mathrm{N}}$ shown in (a) (see the text for definition), as a function of $\kappa_{\text {eff }}$.

\section{CONCLUSIONS}

We conclude by highlighting three results of the present work. The first concerns the repulsive character of pair-interactions between the colloidal platelets of Ref. [30]. The specific interactions between negatively charged platelets partially neutralized with TBA molecules (thus creating surface dipoles) in an aqueous solvent is difficult to model. In particular, hydration layers mediate platelet interactions. The experimental platelet volume fraction for the I-N transition is much higher than that predicted by excluded-volume-based models for given mean aspect ratio, implying the presence of highly repulsive effective interactions between particles. It is then reasonable to map particles onto effective hard-core platelets with a larger effective thickness so as to obtain comparable results between theory and experiment. The effective thickness mainly depends on the di- ameter polydispersity (obviously platelets with large surfaces - their number depending on the width and the tail of the length-distribution function-, and consequently with more charges and dipoles, create much more repulsive effective interactions). Using this procedure, the three experimental samples, separated into three distinct sets A, B, and C (the major difference between them being their polydispersity) were mapped onto effective hard platelets with three different effective thicknesses. The hard model used was based on the restricted-orientation approximation for polydisperse board-like particles and a Fundamental-Measure density functional was used.

Our theoretical results agree with experiment for sets A and B, except for samples with the highest aspect ratios. For set B (samples obtained via fractionation) we used a polydispersity coefficient $\Delta_{0}=0.3$, approximately the same value measured in experiments, and the theory correctly describes the experimental phase behaviour. For samples in set A, agreement is reached for $\Delta_{0} \sim 0.5$, which is higher than the experimental value. However, we have noted (Sec. IIC) that, since colloidal particles are also polydisperse in shape, once we choose the particle geometry the value of the effective polydispersity should be higher. This is in fact the second important result of our work.

The last result is related to the theoretical modeling of samples in set C. We have shown that, in this case, a unimodal size distribution cannot describe the experimental phase behavior correctly as regards the huge density gap of the I-N coexistence and the strong nonlinearity in the percentage of volume occupied by the $\mathrm{N}$ phase as a function of total volume fraction. However, a bimodal size distribution adequately describes the gap and the nonlinearity; note that bimodality may not be apparent by direct visual inspection of the distribution function.

In summary, both fractionation and demixing phenomena are important to explain the experimental results. These conclusions are expected to remain valid even if a more exact theoretical treatment, including free particle orientation, could be implemented.

\section{Appendix A: Free energy following the SPT}

In this section we derive, following the SPT formalism, the expression for the free-energy density of a polydisperse Zwanzig fluid made of hard board-like particles. The fluid consists of a collection of board-like particles with square polydisperse cross-section $\sigma$ and constant thickness $L$. The main particle axes point along one of the three Cartesian axes $x, y, z$. The microscopic variables describing the fluid are the density distribution functions $\rho_{\nu}(\sigma)$, with $\nu$ labeling the different species $(\nu=\{x, y, z\})$.

The work to insert a scaled particle of dimensions $\lambda_{1} \sigma$ (scaled with the width parameter $\lambda_{1}$ ) and $\lambda_{2} L$ (scaled with the thickness parameter $\lambda_{2}$ ) and pointing along the $\nu$ direction in the polydisperse Zwanzig fluid can be cal- 
culated as

$$
\begin{aligned}
& \beta W_{\nu}\left(\lambda_{1}, \lambda_{2}\right)=-\ln \left[1-\sum_{s} \int d \sigma^{\prime} \rho_{s}\left(\sigma^{\prime}\right)\right. \\
& \left.\times V_{\nu}^{(\operatorname{excl})}\left(\lambda_{1} \sigma, \lambda_{2} L, \sigma^{\prime}, L\right)\right]
\end{aligned}
$$

where the excluded volume between the particles $\nu$ and $s$ (the respective labels of particle orientations), the latter with dimensions $\sigma^{\prime} \times \sigma^{\prime} \times L$, has the expression

$$
\begin{aligned}
& V_{\nu s}^{(\mathrm{excl})}\left(\lambda_{1} \sigma, \lambda_{2} L, \sigma^{\prime}, L\right)=\prod_{\tau=\{x, y, z\}}\left[\sigma_{\nu \tau}\left(\lambda_{1} \sigma, \lambda_{2} L\right)\right. \\
& \left.+\sigma_{s \tau}\left(\sigma^{\prime}, L\right)\right] \text {, }
\end{aligned}
$$

where we have defined $\sigma_{\nu \tau}(\sigma, L)=\sigma+(L-\sigma) \delta_{\nu \tau}$, with $\delta_{\nu \tau}$ the Kronecker delta.

Following the SPT, the excess part of the chemical potential of the species $\nu$ can be calculated as a sum of two terms. The first one is the second-order Taylor expansion of $W_{\nu}\left(\lambda_{1}, \lambda_{2}\right)$ around the point $(0,0)$ and evaluated at $(1,1)$. The second one is the product of the fluid pressure $P$ and the particle volume $v=L \sigma^{2}$ (i.e. the thermodynamic work to open a cavity of dimension $v$ ). Thus we have

$$
\begin{aligned}
\mu_{\nu}^{(\mathrm{exc})}(\sigma) & =W_{\nu}(0,0)+\sum_{i} \frac{\partial W_{\nu}(0,0)}{\partial \lambda_{i}} \\
& +\frac{1}{2} \sum_{i, j} \frac{\partial^{2} W_{\nu}(0,0)}{\partial \lambda_{i} \partial \lambda_{j}}+P v
\end{aligned}
$$

Taking into account the thermodynamic relations

$$
P=\sum_{\nu} \int d \sigma \mu_{\nu}(\sigma) \rho_{\nu}(\sigma)-\mathcal{F} / V, \quad \mu_{\nu}(\sigma)=\frac{\delta \mathcal{F} / V}{\delta \rho_{\nu}(\sigma)}
$$

with $\mathcal{F}\left[\left\{\rho_{\nu}\right\}\right], \mu_{\nu}$ and $V$ respectively the free-energy density functional, the chemical potential of species $\nu$ (which is splitted in the ideal and excess part: $\mu_{\nu}(\sigma)=$ $\left.\mu^{(\mathrm{id})}(\sigma)+\mu_{\nu}^{(\mathrm{exc})}(\sigma)\right)$, and the system volume, we obtain the expression for the excess part of the free energy density $\Phi_{\text {exc }} \equiv \beta \mathcal{F}_{\text {exc }} / V$ :

$$
\Phi_{\mathrm{exc}}=-n_{0} \ln \left(1-n_{3}\right)+\frac{\mathbf{n}_{1} \cdot \mathbf{n}_{2}}{1-n_{3}}+\frac{n_{2 x} n_{2 y} n_{2 z}}{\left(1-n_{3}\right)^{2}},
$$

which coincides with the uniform limit of the excess part of the free-energy density for a general inhomogeneous fluid following the FMT [27]. The weighting densities $n_{\alpha}$ are functions of the moments $\rho_{\nu}^{(\alpha)}$ of the distribution function $\rho_{\nu}(\sigma)$ :

$$
\rho_{\nu}^{(\alpha)}=\int d \sigma \rho_{\nu}(\sigma) \sigma^{\alpha}, \quad \alpha=0,1,2
$$

Their expressions are

$$
\begin{aligned}
& n_{0}=\rho^{(0)} \equiv \sum_{\tau} \rho_{\tau}^{(0)}, \quad n_{3}=L \rho^{(2)} \equiv L \sum_{\tau} \rho_{\tau}^{(2)} \\
& n_{1 \nu}=L \rho_{\nu}^{(0)}+\sum_{\tau \neq \nu} \rho_{\tau}^{(1)}, \quad n_{2 \nu}=\rho_{\nu}^{(2)}+L \sum_{\tau \neq \nu} \rho_{\tau}^{(1)}
\end{aligned}
$$

Finally, the pressure can be calculated from (A5) as

$$
\beta P=\frac{\partial \Phi_{\mathrm{exc}}}{\partial n_{3}}=\frac{n_{0}}{1-n_{3}}+\frac{\mathbf{n}_{1} \cdot \mathbf{n}_{2}}{\left(1-n_{3}\right)^{2}}+\frac{2 n_{2 x} n_{2 y} n_{2 z}}{\left(1-n_{3}\right)^{3}} .
$$

\section{Acknowledgments}

We thank Andres Mejia and Zhengdong Cheng for useful and illuminating discussions during the course of the present work and for providing us with their experimental data prior to publication. We also acknowledge financial support from Comunidad Autónoma de Madrid (Spain) under the R\&D Programme of Activities MODELICOCM/S2009ESP-1691, and from MINECO (Spain) under grants MOSAICO, FIS2010-22047-C01 and FIS201022047-C04.
[1] K. R. Purdy, S. Varga, A. Galindo, G. Jackson and S. Fraden, Phys. Rev. Lett. 94, 057801 (2005).

[2] H. Maeda and Y. Maeda, Phys. Rev. Lett. 90, 018303 (2003).

[3] Z. Dogic and S. Fraden, Phys. Rev. Lett. 78, 2417 (1997).

[4] K. R. Purdy and S. Fraden, Phys. Rev. E 76, 011705 (2007).

[5] F. M. van der Kooij, D. van der Beek and H.N. W. Lekkerkerker, J. Phys. Chem. B 105, 1696 (2001).

[6] A. V. Petukhov, D. van der Beek, R. P. A. Dullens, I. P. Dolbnya, G. J. Vroege and H. N. W. Lekkerkerker, Phys. Rev. Lett. 95, 077801 (2005).

[7] D. V. Byelov, M. C. D. Mourad, I. Snigireva, A. Snigirev,
A. V. Petukhov and H. N. W. Lekkerkerker, Langmuir 26, 6898 (2010).

[8] F. M. van der Kooij, K. Kassapidou and H. N. W. Lekkerkerker, Nature 406, 868 (2000).

[9] D. Sun, H.-J. Sue, Z. Cheng, Y. Martínez-Ratón and E. Velasco, Phys. Rev. E 80, 041704 (2009).

[10] D. Kleshchanok, P. Holmqvist, J. M. Meijer, H. N. W. Lekkerkerker, J. Am. Chem. Soc. 134, 5985 (2012).

[11] F. M. van der Kooij and H. N. W. Lekkerkerker, Phys. Rev. Lett. 84, 781 (2000)

[12] E. van den Pol, A. V. Petukhov, D. M. E. Thies-Weesie, D. V. Byelov and G. J. Vroege, Phys. Rev. Lett. 103, 258301 (2009). 
[13] H. H. Wensink, G. J. Vroege and H. N. W. Lekkerkerker, J. Phys. Chem. B 105, 10610 (2001).

[14] A. A. Verhoeff, H. H. Wensink, M. Vis, G. Jackson and H. N. W. Lekkerkerker, J. Phys. Chem. B 113, 13476 (2009).

[15] P. Tarazona, J. A. Cuesta and Y. Martínez-Ratón, Lect. Notes Phys. 753, 247 (2008)

[16] A. Speranza and P. Sollich, J. Chem. Phys. 117, 5421 (2002).

[17] A. Speranza and P. Sollich, J. Chem. Phys. 118, 5213 (2003).

[18] A. Esztermann, H.-Reich and M. Schmidt, Phys. Rev. E 73, 011409 (2006).

[19] M. A. Bates and D. Frenkel, J. Chem. Phys. 110, 6553 (1999).

[20] J. A. C. Veerman and D. Frenkel, Phys. Rev. A 45, 5632 (1992).

[21] M. Marechal, A. Cuetos, B. Martínez-Haya and M. Dijkstra, J. Chem. Phys. 134, 094501 (2011).
[22] R. Zwanzig, J. Chem. Phys. 39, 1714 (1963).

[23] N. Clarke, J. A. Cuesta, R. Sear, P. Sollich and A. Speranza, J. Chem. Phys. 113, 5817 (2000).

[24] Y. Martínez-Ratón and J. A. Cuesta, Phys. Rev. Lett. 89, 185701 (2002).

[25] S. Belli, A. Patti, M. Dijkstra and R. van Roij, Phys. Rev. Lett. 107, 148303 (2011).

[26] Y. Martínez-Ratón, S. Varga and E. Velasco, Phys. Chem. Chem. Phys. 13, 13247 (2011).

[27] J. A. Cuesta and Y. Martínez-Ratón, Phys. Rev. Lett. 78, 3681 (1997).

[28] M. Bier, L. Harnau and S. Dietrich, Phys. Rev. E 69, 021506 (2004).

[29] P. Sollich, P. B. Warren, and M. E. Cates, Adv. Chem. Phys. 116, 265 (2001).

[30] A. F. Mejia, Y.-W. Chang, R. Ng, M. Shuai, M. S. Mannan, and Z. Cheng, Phys. Rev. E 85, 061708 (2012). 